\title{
Producción y calidad del huevo en gallinas en semilibertad alimentadas con diferentes niveles de pasta de canola
}

\section{Performance and egg quality in semi-free range hens fed diets with different levels of canola meal}

\author{
Arturo Cortes Cuevasa, Martha Susana Cedillo Monrroya, Gabriela Gómez Verduzcob, \\ Analía Balderas Gonzáleza, Ernesto Avila Gonzáleza
}

\begin{abstract}
RESUMEN
Se evaluó el comportamiento productivo y calidad organoléptica del huevo, en gallinas Isa-Brown alimentadas con dietas isoproteicas e isocalóricas sorgo-soya con la inclusión de pasta de canola (PC) (0, 6.6, 13.2 y 26.4 \%), como reemplazo de la proteína $(0,25,50$ y $90 \%)$ de pasta de soya (PS). Se realizaron dos experimentos. En el primero se reemplazó el 0, 50 y $90 \%$, se utilizaron 405 gallinas de 23 a 31 semanas de edad, en tres tratamientos con tres repeticiones de 45 gallinas cada una. En el segundo, se reemplazó el 0, 25 y 50 \%, se utilizaron 405 gallinas de 40 a 48 semanas de edad, en tres tratamientos con tres repeticiones de $\mathbf{4 5}$ gallinas cada una. Los resultados en ambos experimentos para porcentaje de postura, peso de huevo, consumo de alimento, masa de huevo, conversión alimenticia, unidades Haugh, color de la yema y grosor de cascarón, indicaron que no existió diferencia ( $P>0.05)$ entre tratamientos. Los datos para sabor del huevo en el Exp 1, mostraron diferencia $(P<0.01)$ con mayor aceptación con 0 y $13.2 \%$ de inclusión y menor aceptación con $26.4 \%$ de PC. En el Exp 2 , el sabor del huevo no difirió $(P>0.05)$ entre tratamientos. Se puede concluir que gallinas Isa Brown alimentadas con dietas sorgoPS, con el reemplazo de 25, 50 y 90 \% de proteína de PS por proteína de PC, no afectó el rendimiento productivo, unidades Haugh y color de la yema; sin embargo, el reemplazo del $90 \%$ (26.4 \% de inclusión de PC), impartió al huevo sabor a pescado.
\end{abstract}

PALABRAS Clave: Pasta de canola, Gallinas Isa Brown, Calidad del huevo, Respuesta productiva, Sabor del huevo.

\begin{abstract}
In order to evaluate performance and organoleptic quality of egg-Isa Brown hens fed sorghum-soybean meal isoproteic and isocaloric diets with different levels of inclusion of canola meal $(0.0,6.6,13.2$ and $26.4 \%)$ as a replacement $(0.0$, 25, 50 and $90 \%$ ) for soybean meal protein (SM), two experiments were conducted. In Exp 1, 13.2 and $26.4 \%$ canola meal levels were used in 405 hens, 23 to 31 wk of age, in three treatments (including a control with $0 \%$ canola meal) with three replicates of 45 hens each. In Exp 2, 6.6 and $13.2 \%$ canola meal levels were used in 405 hens, 40 to 48 wk of age, in three treatments (including a control with $0 \%$ canola meal) with three replicates of 45 hens each. Canola meal inclusion level had no effect $(P>0.05)$ on laying percentage, egg weight, feed intake, egg mass, feed conversion, Haugh units, yolk color and shell thickness in either experiment. Only egg flavor in Exp 1 differed among treatments in that sensory test panelists preferred $(P<0.01)$ eggs from the control and $13.2 \%$ treatments to those in the $\mathbf{2 6 . 4} \%$ treatment. No differences $(P>0.05)$ in organoleptic variables were noted in Exp 2. Replacement of 25,50 and $90 \%$ of protein from soybean meal had no effect on performance or egg quality parameters in ISA Brown hens. However, replacement of $90 \%$ of the protein (26.34 \% inclusion with canola meal) imparted a fishy odor to the eggs.
\end{abstract}

KEY WORDS: Canola meal, ISA Brown hens, Egg quality, Performance, Egg flavor.

Recibido el 7 de julio de 2015. Aceptado el 30 de agosto de 2015.

a Centro de Enseñanza, Investigación y Extensión en Producción Avícola, Facultad de Medicina Veterinaria y Zootecnia, Universidad Nacional Autónoma de México. cortescuevasarturo@yahoo.com. Correspondencia al primer autor.

b Departamento de Producción Animal: Aves, Facultad de Medicina Veterinaria y Zootecnia, Universidad Nacional Autónoma de México. 


\section{INTRODUCCIÓN}

En México la producción de pasta de soya (PS), está determinada por el sector pecuario y depende de la disponibilidad de frijol soya, la cual en México es reducida respecto a la demanda, dando como resultado importación de volúmenes de $97 \%$ contra la producción nacional del $3 \%(1)$. La PS sigue siendo la fuente de proteína por excelencia; sin embargo, es una fuente alternativa de proteína para la alimentación de las aves a niveles bajos, ya que su empleo se limita por la presencia de factores antinutricionales(2). Además de que la canola es más baja en proteína que la PS, la pasta de canola (PC) contiene mayor cantidad de metionina y cistina, pero menor contenido de lisina; sin embargo, ambas pastas se complementan una a la otra al emplearlas en la formulación de raciones(3).

Entre los factores anti-nutricionales que afectaban el valor nutrimental de la pasta de nabo, antecedente de las PC actuales, están el ácido erúsico y glucosinolatos, con efectos negativos en el comportamiento productivo: las nuevas variedades de semillas de PC llamadas doble " 00 " presentan valores más bajos de glucosinolatos (< a $20 \mu \mathrm{g} / \mathrm{g}$ ) y ácido erúsico (cantidades traza) $(2,4,5)$.

En estudios realizados por Ciuresco(6) en gallinas, determinaron que la PC puede ser empleada en las dietas hasta un $20 \%$ para reemplazar del 25 a $30 \%$ de pasta de soya, sin afectar el rendimiento productivo y la calidad del huevo. Así mismo, en una evaluación sensorial(7), los panelistas detectaron en huevos crudos la incidencia de olor a pescado en huevos marrón en $28 \%$ (rango 15-40) y en huevo blanco el $18 \%$ (rango 10-30), cuando las gallinas se alimentaron con 12 y $20 \%$ de PC en el alimento. La prueba con huevos cocidos mostró que huevos de cascarón marrón fueron más altos en la intensidad del olor respecto a los controles y huevos de cascarón blancos.

Perez-Maldonado et $a(8)$ usando 10,15 y $20 \%$ de PC en la dieta en gallinas Isa-Brown, no

\section{INTRODUCTION}

Soybean meal (Glycine max) is a widely used ingredient in livestock feed for its excellent protein content. Its production in Mexico is for use in the livestock industry, and is limited by the small scale of domestic soy bean production. Only $3 \%$ of soybean meal demand is met by domestic production, the remaining $97 \%$ coming from imported raw material(1). Despite its excellent protein content, its antinutritional factor content means soybean meal can only be used as an alternative protein source at low levels in poultry diets(2). Often, it is used together with canola (Brassica rapa) meal since they complement each other's nutritional properties. Canola meal has lower protein and lysine contents than soybean meal, but higher methionine and cysteine contents(3).

Antinutritional factors have been an issue in development of canola. Rapeseed (Brassica napus) is the parent species to canola, but has not been widely used in livestock applications due to its erusic acid and glucosinolates content, which negatively affect productive performance. Modifications to canola created the new " 00 " varieties, so called for their extremely low glucosinolate content $(<20 \mu \mathrm{g} / \mathrm{g})$ and trace erusic acid content $(2,4,5)$.

Canola meal has been used successfully in diets for hens at up to $20 \%$ to replace from 25 to $30 \%$ of soybean meal, without affecting egg yield or quality $(6)$. However, sensory evaluations of eggs from hens fed diets containing 12 or $20 \%$ canola meal identified a fishy odor in $28 \%$ (range $=15-40$ ) of raw brown eggs and $18 \%$ (range 10-30) of raw white eggs(7). When cooked, the odor in the brown eggs was noticeably more intense than in a control treatment or in white eggs. In a study of canola meal $(10,15$ and $20 \%)$ used in diets for ISA Brown hens, no effects were observed on egg production or animal mortality(8). A fishy odor was present, however, at the 15 and $20 \%$ substitution levels, but diminished after storage at $10^{\circ} \mathrm{C}$ for two to five weeks. No differences 
observaron efecto en la producción de huevo y mortalidad; en los tratamientos con 15 y $20 \%$ de PC, los huevos tuvieron olor a pescado en fresco, sin embargo el olor disminuyó cuando los huevos se almacenaron a $10^{\circ} \mathrm{C}$ por 2 a 5 semanas. En cuanto a la evaluación sensorial no encontraron diferencias. El olor a pescado se debe a la presencia de sinapina en la PC; este compuesto presenta problema en un porcentaje pequeño en gallinas semipesadas, debido a que carecen de la enzima trimetil amino oxidasa para degradar este compuesto y evitar que se deposite en el huevo(2).

Al evaluar en gallinas Isa Brown el empleo de 8 y $10 \%$ de PC(9), los datos mostraron reducción en el peso de los huevos (62.9, $61.8,60.7 \mathrm{~g}$ ), el peso de la yema fue mayor respecto al de la albúmina en las aves alimentadas con $20 \%$ de PC; las unidades Haugh, resistencia y grosor de cascarón no se vieron afectados. En el análisis sensorial, el sabor y la aceptabilidad fueron menores en los huevos de gallinas con PC. Se ha demostrado(10) que la adición de $10 \%$ de PC en las dietas, aumentó el peso del cascarón pero el de la yema disminuyó; además, no se observaron diferencias en unidades Haugh, grosor de la cáscara y resistencia de la cáscara. En otro trabajo(11) dietas con $0,4,6$ y $8 \%$ de PC, no mostraron diferencias en el rendimiento productivo y calidad interna y externa del huevo.

Con estos antecedentes, el presente estudio se planteó para evaluar el empleo de PC (6.6, 13.2 y $26.4 \%$ ), en dietas sorgo-soya para gallinas de postura Isa Brown en semilibertad y su efecto en el rendimiento productivo, pigmentación de la yema y la calidad organoléptica.

\section{MATERIAL Y MÉTODOS}

Se realizaron dos experimentos, con gallinas de la estirpe Isa Brown en un sistema de producción en semilibertad. Ambos experimentos en el trabajo de campo tuvieron una duración de 56 días, y se utilizaron casetas between treatments were observed in the sensory analysis. Presence of the alkaloidal amine sinapine in canola is the cause of the fishy odor. It is problematic in a small percentage of semi-heavy hens that lack the enzyme trimethyl amino oxidase needed to degrade this compound and prevent its deposition in the egg(2).

Use of canola meal in laying hen diets has been shown to affect egg quality parameters. In a study of 8,10 and $20 \%$ inclusion levels of canola paste in diets for ISA Brown hens, overall egg weight was found to decrease (62.9, $61.8,60.7 \mathrm{~g}$, respectively), and yolk weight was greater than albumin weight at the $20 \%$ level( $(9)$. Values for Haugh units (HU), and shell strength and thickness were unaffected. Sensory analysis in the same study found egg flavor and acceptability to be lower in the canola meal treatments. Use of $10 \%$ canola meal in diets for hens was reported to increase shell weight and lower yolk weight, but had no effects on Haugh units, or shell thickness and resistance(10). Lower canola meal inclusion levels $(0,4,6$ and $8 \%)$ produced no effects on egg production yield and internal and external quality characteristics(11).

The present study objective was to evaluate the effect of three canola meal inclusion levels $(6.6,13.2$ and $26.4 \%)$ in sorghum-soya based diets for semi-free range ISA Brown laying hens on egg production yield, yolk pigmentation and organoleptic quality.

\section{MATERIAL AND METHODS}

Two experiments were run using ISA Brown hens in a semi-free range production system. Animals were housed in experimental pens in a natural environment at the Center for Poultry Production Teaching, Research and Outreach (Centro de Enseñanza, Investigación y Extensión en Producción Avícola) at the National Autonomous University of Mexico (Universidad Nacional Autónoma de México - UNAM). Each corral was divided into two sections, one 
experimentales de ambiente natural, localizadas dentro del Centro de Enseñanza, Investigación y Extensión en Producción Avícola de la Universidad Nacional Autónoma de México, dividiendo cada caseta en dos secciones, con cama de paja y salida a un área de pasto. El acceso al pastoreo fue restringido de 0900 a 1200 durante todo el experimento. Cada corral contó con cuatro comederos de tolva y dos bebederos de campana. Se les proporcionó un fotoperiodo de 16 h luz x día. La alimentación containing straw beds and the other an open area with grass. Access to the grass area was restricted to 0900 to 1200 throughout the experimental period. Each pen contained four feeders and two bell drinkers. Photoperiod was $16 \mathrm{~h}$ light: $8 \mathrm{~h}$ dark. Both feed and water were made freely available throughout the experimental period.

Both experiments involved 405 red semi-heavy ISA Brown laying hens. Exp 1 lasted for $49 \mathrm{~d}$

Cuadro 1. Composición de las dietas para gallinas Isa Brown con diferentes niveles de canola (Exp 1)

Table 1. Composition of diets containing different levels of canola meal as a replacement for soybean protein fed ISA Brown hens (Exp 1)

\begin{tabular}{lccc}
\hline & Canola 0 \% & Canola 13.2 \% & Canola 26.4 \% \\
\hline Sorghum & 634.311 & 586.794 & 539.205 \\
Soybean meal & 215.457 & 116.140 & 16.673 \\
Canola meal & - & 131.900 & 264.00 \\
Calcium carbonate & 105.191 & 103.733 & 102.272 \\
Vegetable oil & 17.778 & 35.056 & 52.360 \\
Monocalcium phosphate & 11.315 & 10.514 & 9.712 \\
Salt & 4.658 & 4.414 & 4.169 \\
Vitamins and minerals & 2.500 & 2.500 & 2.500 \\
Methionine 88\% & 2.370 & 1.891 & 1.411 \\
Mycotoxin sequestrant & 2.000 & 2.000 & 2.000 \\
L-lysine HCl & 1.429 & 2.046 & 2.664 \\
Yellow pigment (Tagetes) & 1.000 & 1.000 & 1.000 \\
Red pigment (Capsicum) & 0.800 & 0.800 & 0.800 \\
Choline chloride 60\% & 0.500 & 0.500 & 0.500 \\
Zinc bacitracin & 0.300 & 0.300 & 0.300 \\
L-threonine & 0.189 & 0.211 & 0.234 \\
Antioxidant & 0.100 & 0.100 & 0.100 \\
Phytase & 0.100 & 0.100 & 0.100 \\
Nutrients analysis & & & \\
Protein, \% & 16.000 & 16.000 & 16.000 \\
ME, Kcal/kg & 2.750 & 2.750 & 2.750 \\
Total calcium, \% & 4.100 & 4.100 & 4.100 \\
Sodium, \% & 0.190 & 0.190 & 0.190 \\
Available phosphorous, \% & 0.440 & 0.440 & 0.440 \\
Met + Cyst Dig, \% & 0.629 & 0.623 & 0.616 \\
Lysine Dig, \% & 0.797 & 0.769 & 0.741 \\
Threonine Dig, \% & 0.527 & 0.515 & 0.502 \\
\hline
\end{tabular}

$\mathrm{ME}=$ metabolizable energy; * Vitamin A 10,000,000 IU; Vitamin D3 2,500,000 IU; Vitamin E 10, $000 \mathrm{IU}$; Vitamin K $2.5 \mathrm{~g}$; thiamin $1.6 \mathrm{~g}$; riboflavin $5 \mathrm{~g}$; cyanocobalamin $0.010 \mathrm{~g}$, folic acid 0.50 g; pyridoxine $1.5 \mathrm{~g}$; calcium pantothenate $10 \mathrm{~g}$; niacin $30 \mathrm{~g}$; choline chloride $60 \% 200 \mathrm{~g}$; iron 40 $\mathrm{g}$; manganese $80 \mathrm{~g}$; copper $10 \mathrm{~g}$; iodine $2 \mathrm{~g}$; zinc $60 \mathrm{~g}$; selenium $0.30 \mathrm{~g}$. 
y el agua se proporcionaron ad libitum durante todo el estudio.

En los experimentos, se utilizaron 405 gallinas rojas semipesadas en semilibertad de la estirpe Isa-Brown de 30 y 45 semanas de edad respectivamente. En cada experimento se empleó un diseño completamente al azar con tres tratamientos cada uno con tres réplicas de 45 gallinas. Las aves en ambos experimentos se alojaron en corrales, con espacio de $0.73 \mathrm{~m}^{2}$ and hens began the experiment at $30 \mathrm{wk}$ of age. Exp 2 lasted for $56 \mathrm{~d}$ and hens began at $45 \mathrm{wk}$ of age. In each experiment, the design was completely random, with three treatments including three replicates of 45 animals each. The animals were housed in pens with an enclosed area providing $0.73 \mathrm{~m}^{2}$ per animal and a grazing area providing $1.3 \mathrm{~m}^{2}$ per animal.

Diets were formulated using sorghum and soybean meal (SP), with different canola meal

Cuadro 2. Composición de las dietas para gallinas Isa Brown con diferentes niveles de canola (Exp 2)

Table 2. Composition of diets containing different levels of canola meal as a replacement for soybean protein fed ISA Brown hens (Exp 2)

\begin{tabular}{lccc}
\hline & Canola 0 \% & Canola 6.6 \% & Canola 13.2 \% \\
\hline Sorghum & 634.311 & 614.166 & 586.794 \\
Soybean meal & 215.457 & 159.610 & 116.140 \\
Canola meal & - & 65.950 & 131.900 \\
Calcium carbonate & 105.191 & 104.471 & 103.733 \\
Vegetable oil & 17.778 & 26.830 & 35.056 \\
Monocalcium phosphate & 11.315 & 10.956 & 10.514 \\
Salt & 4.658 & 4.538 & 4.414 \\
Vitamins and minerals* & 2.500 & 2.500 & 2.500 \\
Methionine 88\% & 2.370 & 3.222 & 1.891 \\
Mycotoxin sequestrant & 2.000 & 2.000 & 2.000 \\
L-lysine HCl & 1.429 & 2.302 & 2.046 \\
Yellow pigment (Tagetes) & 1.000 & 1.000 & 1.000 \\
Red pigment (Capsicum) & 0.800 & 0.800 & 0.800 \\
Choline chloride 60\% & 0.500 & 0.500 & 0.500 \\
Zinc bacitracin & 0.300 & 0.300 & 0.300 \\
L-threonine & 0.189 & 0.605 & 0.211 \\
Antioxidant & 0.100 & 0.150 & 0.100 \\
Phytase & 0.100 & 0.100 & 0.100 \\
Nutrients analysis & & & \\
Protein, \% & 16.000 & 16.000 & 16.000 \\
ME, Kcal/kg & 2.750 & 2.750 & 2.750 \\
Total calcium, \% & 4.100 & 4.100 & 4.100 \\
Sodium, \% & 0.190 & 0.190 & 0.190 \\
Available phosphorous, \% & 0.440 & 0.440 & 0.440 \\
Met + Cyst Dig, \% & 0.629 & 0.705 & 0.623 \\
Lysine Dig, \% & 0.797 & 0.794 & 0.769 \\
Threonine Dig, \% & 0.527 & 0.541 & 0.515 \\
\hline
\end{tabular}

$\mathrm{ME}=$ metabolizable energy; ${ }^{*}$ Vitamin A 10,000,000 IU; Vitamin D3 2,500,000 IU; Vitamin E 10, 000 IU; Vitamin K $2.5 \mathrm{~g}$; thiamin $1.6 \mathrm{~g}$; riboflavin $5 \mathrm{~g}$; cyanocobalamin $0.010 \mathrm{~g}$, folic acid $0.50 \mathrm{~g}$; pyridoxine $1.5 \mathrm{~g}$; calcium pantothenate $10 \mathrm{~g}$; niacin $30 \mathrm{~g}$; choline chloride $60 \% 200 \mathrm{~g}$; iron $40 \mathrm{~g}$; manganese 80 $\mathrm{g}$; copper $10 \mathrm{~g}$; iodine $2 \mathrm{~g}$; zinc $60 \mathrm{~g}$; selenium $0.30 \mathrm{~g}$. 
por ave y en los espacios de pastoreo de $1.3 \mathrm{~m}^{2}$ por ave. Los tratamientos experimentales consistieron:

\section{Experimento 1:}

Tratamiento 1) Dieta sorgo-soya (EM= 2,750 $\mathrm{kcal} / \mathrm{kg}, 16 \% \mathrm{PC})$.

Tratamiento 2) Dieta sorgo-soya $+13.2 \%$ de $P C(E M=2,750 \mathrm{kcal} / \mathrm{kg}, 16 \% \mathrm{PC})$.

Tratamiento 3) Dieta sorgo-soya $+26.4 \%$ de $\mathrm{PC}(\mathrm{EM}=2,750 \mathrm{kcal} / \mathrm{kg}, 16 \% \mathrm{PC})$.

\section{Experimento 2:}

Tratamiento 1) Dieta sorgo-soya (EM= 2,750 $\mathrm{kcal} / \mathrm{kg}, 16 \% \mathrm{PC})$.

Tratamiento 2) Dieta sorgo-soya + $6.6 \% \mathrm{PC}$ $(E M=2,750 \mathrm{kcal} / \mathrm{kg}, 16 \% \mathrm{PC})$.

Tratamiento 3) Dieta sorgo-soya $+13.2 \%$ de $P C(E M=2,750 \mathrm{kcal} / \mathrm{kg}, 16 \% \mathrm{PC})$.

El reemplazo de proteína de pasta de soya (25, 50 y $90 \%$ ), se hizo por proteína de PC, lo que representó el empleo de $6.6,13.2$ y $26.4 \%$ de PC. La composición nutrimental de las dietas de los experimentos 1 y 2 , se pueden observar en los Cuadros 1 y 2 respectivamente.

En los dos experimentos se llevaron registros semanales de porcentaje de postura, peso promedio de huevo, masa del huevo, consumo de alimento, conversión alimenticia y porcentaje de mortalidad. Al final de cada experimento, se tomaron 30 huevos por tratamiento para evaluar su calidad por medio de las variables unidades Haugh, grosor del cascarón y la pigmentación de la yema con el abanico colorimétrico de DSM.

También se llevaron a cabo análisis organolépticos del huevo; se tomaron 10 huevos por tratamiento al Instituto Nacional de Ciencias Médicas y Nutrición Salvador Zubirán, en el Área de Nutrición animal, en la Delegación Tlalpan, substitution levels (Tables 1, 2). Replacement of SP was done based on percentage of protein content (25, 50 and 90 \%). Based on canola protein content, the resulting substitution levels were $6.6,13.2$ and $26.4 \%$ canola. The treatments in each experiment were as follows:

\section{Experiment 1:}

Treatment 1) Sorghum-soya diet $(\mathrm{ME}=2,750$ $\mathrm{kcal} / \mathrm{kg}, 16 \% \mathrm{CP}$ ).

Treatment 2) Sorghum-soya diet $+13.2 \%$ canola $(\mathrm{ME}=2,750 \mathrm{kcal} / \mathrm{kg}, 16 \% \mathrm{CP})$.

Treatment 3) Sorghum-soya diet $+26.4 \%$ canola $(\mathrm{ME}=2,750 \mathrm{kcal} / \mathrm{kg}, 16 \% \mathrm{CP})$.

\section{Experiment 2:}

Treatment 1) Sorghum-soya diet $(\mathrm{ME}=2,750$ $\mathrm{kcal} / \mathrm{kg}, 16 \% \mathrm{CP}$ ).

Treatment 2) Sorghum-soya diet $+6.6 \%$ canola $(\mathrm{ME}=2,750 \mathrm{kcal} / \mathrm{kg}, 16 \% \mathrm{CP})$.

Treatment 3) Sorghum-soya diet $+13.2 \%$ canola (ME $=2,750 \mathrm{kcal} / \mathrm{kg}, 16 \% \mathrm{CP}$ ).

Weekly data were collected on laying percentage, average egg weight, egg mass, feed intake, feed conversion and percentage mortality. At the end of each experiment, 30 eggs per treatment were collected to evaluate quality characteristics, including Haugh Units, shell thickness, and yolk pigmentation (using the DSM colorimetric fan).

Organoleptic evaluations were also made of the eggs. Ten eggs per treatment were taken to the Animal Nutrition Area of the Salvador Zubirán National Institute of Medical and Nutrition Sciences (Instituto Nacional de Ciencias Médicas y Nutrición Salvador Zubirán), Mexico City. Tests were run using 29 untrained panelists who confirmed they were regular egg eaters. They evaluated the eggs while seated in individual cubicles under white light, in the institute's sensory evaluation laboratory. Panelists expressed their like or dislike of yolk color and 
D.F. Las pruebas se llevaron a cabo en cubículos individuales con luz blanca, en el laboratorio de evaluación sensorial de este instituto. Participaron 29 panelistas no entrenados, consumidores de huevo habitual. La evaluación consistió en cuánto agrada o desagrada el color de yema y sabor del huevo, utilizándose dos cuestionarios para cada panelista, donde se mostraron escalas categorizadas. Para la evaluación, cada categoría se convirtió en puntajes del 1 al 5 , en donde 5 gustó mucho, 4 gustó poco, 3 indiferente, 2 disgustó poco y 1 disgustó mucho. Para evaluar el color de yema se presentó a cada panelista una charola con cuatro moldes transparentes con una yema de huevo, de cada tratamiento y su cuestionario respectivo. A continuación se les presentó un plato con tres diferentes muestras de huevo cocinado (revuelto sin aceite y sin sal), acompañados con pan blanco y agua, que consumieron antes de probar cada muestra, evaluando así el sabor del huevo. Al último se les pidió el cuestionario contestado, y a los datos obtenidos se les aplicó la prueba de Friedman del paquete estadístico SPSS(12).

A cada una de las variables de la respuesta productiva en ambos experimentos, se les realizó un análisis de varianza conforme a un diseño completamente al azar, y cuando existieron diferencias estadísticas $(P<0.05)$ entre tratamientos, se realizó una comparación de medias mediante la prueba de Tukey del paquete estadístico SPSS(12). egg flavor using two questionnaires of categorized scales per panelist. Each category was divided into scores of 1 to $5: 5=$ like very much; 4= like; $3=$ indifferent; 2 dislike; $1=$ dislike very much. Yolk color was evaluated by presenting each panelist with a tray (corresponding to a treatment) of four transparent molds each containing a yolk, and the corresponding questionnaire. Egg flavor was evaluated by presenting the panelists with a plate containing three samples of egg prepared by cooking scrambled without salt or oil. They were also given white bread and water to eat after each tasting. Questionnaire data was entered into a database and a Friedman test applied to them with the SPSS statistics package(12).

Production behavior data from both experiments were processed with a completely random ANOVA, and a Tukey test to compare the means of statistically different $(P<0.05)$ treatments. All statistical analyses were done with the SPSS statistics package(12).

\section{RESULTS}

Performance variables (i.e. laying percentage, egg weight, feed intake, egg mass and feed conversion) did not differ ( $P>0.05$ ) among treatments (Tables 3, 4). The same held true for the egg quality variables (Haugh Units, yolk pigmentation and shell thickness), with no differences ( $P>0.05)$ observed among treatments (Tables 5, 6).

Cuadro 3. Resultados a los 49 días de experimentación con dietas elaboradas a diferentes niveles de inclusión de canola $(\operatorname{Exp} 1)^{*}$

Table 3. Performance at end of $49-d$ period of feeding with diets containing different levels of canola meal as a replacement for soybean protein (Exp 1)*

\begin{tabular}{lccccc}
\hline Treatment & Laying $(\%)$ & Egg weight $(\mathrm{g})$ & Feed intake $(\mathrm{g})$ & Egg mass $(\mathrm{g})$ & Feed conversion \\
\hline Canola 0\% & $94.7 \pm 0.48$ & $60.1 \pm 0.24$ & $121 \pm 1.68$ & $56.9 \pm 0.40$ & $2.007 \pm 0.02$ \\
Canola 13.2\% & $95.3 \pm 0.23$ & $59.6 \pm 0.20$ & $120 \pm 0.60$ & $57.1 \pm 0.24$ & $2.013 \pm 0.01$ \\
Canola 26.4\% & $92.4 \pm 0.44$ & $59.5 \pm 0.29$ & $121 \pm 0.64$ & $55.2 \pm 0.26$ & $2.031 \pm 0.01$ \\
\hline
\end{tabular}

* Average of mean \pm standard error; $(P>0.05)$. 
Cuadro 4. Resultados a los 56 días de experimentación con dietas elaboradas a diferentes niveles de inclusión de canola $(\operatorname{Exp} 2)^{*}$

Table 4. Performance at end of $56-\mathrm{d}$ period of feeding with diets containing different levels of canola meal as a replacement for soybean protein $(\operatorname{Exp} 2)^{*}$

\begin{tabular}{lccccc}
\hline Treatment & Laying $(\%)$ & Egg weight $(\mathrm{g})$ & Feed intake $(\mathrm{g})$ & Egg mass $(\mathrm{g})$ & Feed conversion \\
\hline Canola 0 \% & $93.0 \pm 0.41$ & $62.0 \pm 0.10$ & $123.0 \pm 1.14$ & $57.7 \pm 0.25$ & $1.983 \pm 0.02$ \\
Canola 6.6\% & $89.9 \pm 0.34$ & $61.1 \pm 0.10$ & $120.8 \pm 1.42$ & $54.9 \pm 0.25$ & $1.991 \pm 0.02$ \\
Canola 13.2\% & $91.4 \pm 0.50$ & $61.1 \pm 0.14$ & $123.2 \pm 1.44$ & $55.8 \pm 0.37$ & $2.005 \pm 0.03$ \\
\hline
\end{tabular}

* Average of mean \pm standard error. $(P>0.05)$.

\section{RESULTADOS}

Los resultados promedio para porcentaje de postura, peso del huevo, consumo de alimento, masa de huevo y conversión alimenticia en los Exp 1 y 2 se pueden apreciar en los Cuadros 3 y 4 respectivamente. Se observa que los datos de cada una de las variables evaluadas fueron similares $(P>0.05)$ entre tratamientos cuando
In contrast, some differences between treatments were observed in the sensory tests. In Exp 1, yolk color did not differ $(P>0.05)$ between treatments, but egg flavor in the 0 and $13.2 \%$ canola treatments was more widely accepted $(P<0.01)$ than in the $26.4 \%$ canola treatment (Table 7). For Exp 2, no differences $(P>0.05)$ were observed for either variable in either of the canola treatments (6.6 and $13.2 \%$ ) (Table 8).

Cuadro 5. Resultados a los 49 días de experimentación para la calidad de huevo. (Exp 1)*

Table 5. Egg quality results at end of 49-d period of feeding with diets containing different levels of canola meal $(\operatorname{Exp} 1)^{*}$

\begin{tabular}{lccc}
\hline Treatment & Haugh units & Yolk pigmentation $^{* *}$ & Shell thickness $(\mu \mathrm{m})$ \\
\hline Canola 0 \% & $94.3 \pm 0.95$ & $10.66 \pm 0.14$ & $375.1 \pm 4.52$ \\
Canola 13.2 \% & $91.3 \pm 1.66$ & $9.73 \pm 0.10$ & $372.4 \pm 4.21$ \\
Canola 26.4 \% & $93.8 \pm 0.96$ & $9.90 \pm 0.10$ & $364.5 \pm 3.07$ \\
\hline
\end{tabular}

* Average of mean \pm standard error. $(P>0.05)$.

${ }^{* *}$ Data from DSM yolk colorimeter fan.

Cuadro 6. Resultados a los 56 días de experimentación para la calidad de huevo. (Exp 2)

Table 6. Egg quality results at end of 56-d period of feeding with diets containing different levels of canola meal $(\operatorname{Exp} 2)^{*}$

\begin{tabular}{lccc}
\hline Treatment & Haugh units & Yolk pigmentation $^{* *}$ & Shell thickness $(\mu \mathrm{m})$ \\
\hline Canola 0 \% & $88.07 \pm 1.64$ & $9.93 \pm 0.13$ & $380 \pm 3.85$ \\
Canola 6.6 \% & $86.64 \pm 1.83$ & $10.00 \pm 0.10$ & $369 \pm 3.90$ \\
Canola 13.2\% & $88.16 \pm 1.40$ & $10.00 \pm 0.32$ & $372.2 \pm 4.90$ \\
\hline
\end{tabular}

* Average of mean \pm standard error $(P>0.05)$.

${ }^{* *}$ Data from DSM yolk colorimeter fan. 
las gallinas consumieron dietas con $0,6.6,13.2$ y $26.4 \%$ de PC.

Los resultados de las variables unidades Haugh, pigmentación de la yema y grosor de cascarón de los Exp 1 y 2 se muestran en los Cuadros 5 y 6 respectivamente, donde se puede observar que los datos fueron similares entre tratamientos, indicando que con 0,25 , 50 y $90 \%$ de reemplazo de proteína de pasta de soya por proteína de PC, representa el empleo de $6.6,13.2$ y $26.4 \%$, no mostrando diferencias $(P>0.05)$ entre tratamientos para estas variables.

Los datos promedio obtenidos de la prueba sensorial para color de la yema y sabor del huevo se pueden apreciar en los Cuadros $7 \mathrm{y}$ 8 de los $\operatorname{Exp} 1$ y 2 respectivamente. Los resultados del Exp 1, indicaron que no hubo diferencia $(P>0.05)$ entre tratamientos en la percepción del color; sin embargo, hubo una mayor aceptación del sabor en los tratamientos con 0 y $13.2 \%$ de $P C$ respecto al tratamiento con $26.4 \%$ de PC $(P<0.01)$. Los resultados de estas mismas variables en el Exp 2 en la aceptación del color de la yema y aceptación del sabor del huevo no existió diferencia $(P>0.05)$ entre los tratamientos con 6.6 y $13.2 \%$ de PC.

Cuadro 7. Resultados promedio de la prueba sensorial para color y sabor del huevo (Exp 1)

Table 7. Sensory test results for yolk color and egg flavor of eggs $(\operatorname{Exp} 1)^{*}$

\begin{tabular}{lll}
\hline Treatment & Color & Flavor \\
\hline Canola 0\% & $4 \pm 0.2$ a & $4 \pm 0.1$ a \\
Canola 13.2 \% & $4 \pm 0.1$ a & $5 \pm 0.1$ a \\
Canola 26.4 \% & $4 \pm 0.2$ a & $3 \pm 0.2 \mathrm{~b}$ \\
\hline
\end{tabular}

* Average of mean \pm standard error. $(P>0.05)$.

ab Different superscripts in columns indicate difference $(P<0.01)$.

Scale: $1=$ strong dislike, $2=$ mild dislike, $3=$ indifferent, $4=$ like somewhat, and $5=$ like a lot.

\section{DISCUSSION}

Addition of canola at different concentrations $(6.6,13.2$ and $26.4 \%)$ to the sorghum-soya meal diet had no effect on productive parameters. This coincides with previous studies such as one evaluating Dekalb SCWL hens fed a wheat-soya diet with 10 or $20 \%$ added canola, in which neither egg production nor animal mortality were affected(13). However, feed intake did decrease (102.2 vs $99.8 \mathrm{~g}$ ) in the $20 \%$ canola treatment. In another study involving Bovans Brown hens fed corn-soya diets with 4,6 or $8 \%$ added canola, no effect $(P>0.05)$ was observed on performance compared to a control(11).

Other studies using ISA Brown hens have reported variations in some parameters. Using corn-soya diets with 8 or $10 \%$ added canola one study found no differences in laying percentage but egg weight dropped significantly in the $10 \%$ canola treatment(14). Addition of salt $(3 \mathrm{mg} / \mathrm{kg}$ feed) to the $10 \%$ canola treatment somewhat improved egg weight compared to the $10 \%$ canola containing no added salt (62.1 vs $60.7 \mathrm{~g})$. In another study using ISA Brown hens, addition of 10,15 or $20 \%$ canola to sorghum-soya diets had no effect on egg production, although average egg weight did decrease $(P<0.05)$ in the 15 and $20 \%$ treatments $(8)$.

Much more variable results than observed in the present study have been reported elsewhere.

Cuadro 8. Resultados promedio de la prueba sensorial para color y sabor del huevo (Exp 2)*

Table 8. Sensory test results for yolk color and egg flavor of eggs (Exp 2)*

\begin{tabular}{lcc}
\hline Treatment & Color & Flavor \\
\hline Canola 0 \% & $4.0 \pm 0.14$ & $4.0 \pm 0.25$ \\
Canola 6.6 \% & $4.0 \pm 0.17$ & $4.0 \pm 0.20$ \\
Canola 13.2 \% & $4.0 \pm 0.21$ & $3.0 \pm 0.24$ \\
\hline
\end{tabular}

* Average of mean \pm standard error. $(P>0.05)$.

Scale: $1=$ strong dislike, $2=$ mild dislike, $3=$ indifferent, $4=$ like somewhat, and $5=$ like a lot. 


\section{DISCUSIÓN}

Los datos de parámetros productivos en ambos experimentos mostraron que no existió diferencia $(P>0.05)$ a la adición de 6.6, 13.2 y $26.4 \%$ de PC en dietas sorgo-soya. Estos resultados coinciden con los obtenidos por otros autores(13), quienes al realizar un estudio en gallinas Dekalb SCWL, incluyeron 10 y $20 \%$ de pasta de canola en dietas a base de trigo-soya, y encontraron que la producción de huevo y mortalidad no se vieron afectadas; sin embargo, el consumo de alimento se redujo (102.2 vs $99.8 \mathrm{~g}$ ) en el tratamiento con $20 \%$ de inclusión. Swiatkiewicz et a(11), realizaron un estudio en gallinas Bovans Brown utilizando dietas maízsoya, donde incluyeron $0,4,6$ y $8 \%$ de PC, estos investigadores concluyeron que ningún nivel de inclusión tuvo efecto significativo ( $P>0.05)$ sobre los parámetros productivos en comparación con las gallinas alimentadas con la dieta control.

Lichounikova et $a)^{(14)}$ investigaron en gallinas Isa-Brown el empleo de 8 y $10 \%$ de PC en dietas maíz-soya y encontraron resultados similares en porcentaje de postura; no así en peso del huevo, el cual se redujo significativamente en las aves alimentadas con $10 \%$ de PC. Estos autores al adicionar $3 \mathrm{mg} / \mathrm{kg}$ de alimento de yodo a una dieta con $10 \%$ de pasta de canola, encontraron una mejoría en el peso del huevo $(62.1$ vs $60.7 \mathrm{~g})$ respecto a la misma dieta que no fue adicionada con este mineral. También se reporta(8) que empleando 10,15 y $20 \%$ de PC en dietas sorgo-soya para gallinas Isa Brown no se afectó la producción de huevo, pero el peso promedio del huevo fue menor $(P<0.05)$ en las aves que consumieron las dietas con 15 y $20 \%$.

Otros investigadores, han observado resultados diferentes a los obtenidos en el presente estudio. En gallinas Leghorn, el empleo de 0, 5, 10, y $15 \%$ de PC en dietas sorgo-soya, se observó que se afectó $(P<0.05)$ el porcentaje de postura y el peso del huevo en las gallinas con $15 \%$ de inclusión(15). Olomu et a(16), emplearon 0 ,
In a study of addition of 5,10 or $15 \%$ canola to sorghum-soya diets fed Leghorn hens, the $15 \%$ treatment was found to negatively affect $(P<0.05)$ laying percentage and egg weight(15). Another study using Leghorn hens fed diets containing $5,7.5$ or $10 \%$ non-genetically modified canola showed that egg production dropped $(P<0.05)$ in the $10 \%$ treatment, although egg weight exhibited no changes.

In both Exp 1 and Exp 2, Haugh Units, yolk pigmentation and shell thickness values did not vary $(P>0.05)$ among treatments. This agrees with other studies in which addition of 5, 7.5 or $10 \%$ non-genetically modified canola had no effect on Haugh Units values(16), and addition of 5,10 or $15 \%$ canola did not affect Haugh Units values or shell thickness(14). In a study using Hy-Line W36 hens fed diets containing 5, 10 or $15 \%$ canola, no effect was observed on Haugh Units values or shell thickness(10).

Unlike the productive parameters, egg flavor clearly varied between treatments in $\operatorname{Exp} 1$ : the control and $13.2 \%$ canola treatment were preferred $(P<0.01)$ to the $26.4 \%$ canola treatment. This coincides partially with egg flavor results in a study of Bovans Brown hens fed diets containing 4,6 or $8 \%$ canola in which the $8 \%$ canola treatment eggs had inferior $(P<0.05)$ flavor compared to the other treatments(11). Using boiled eggs from hens fed diets containing 8 or $10 \%$ canola, another study found differences in flavor and aroma between the control treatment and the canola treatments(14). The fishy odor that lowers consumer acceptance was also notable in cooked and raw eggs from $\mathrm{Hy}$-line Brown hens fed diets including 12 or $20 \%$ canola(7); the aroma was less pronounced in cooked eggs than in raw ones.

Sinapine is known to be the primary form of choline in canola, and is a precursor of trimethylamine, which can produce the fishy odor and flavor in eggs from semi-heavy hens fed diets containing canola $(17,18,19)$. In an effort 
$5,7.5$ y $10 \%$ de PC no mejorada genéticamente en gallinas Leghorn, y demostraron que la producción de huevo se afectó $(P<0.05)$ al incluir $10 \%$ de esta pasta, sin embargo el peso del huevo no se disminuyó.

En ambos experimentos, las unidades Haugh, la pigmentación de la yema y el grosor del cascarón fueron similares $(P>0.05)$ entre tratamientos, resultados que coinciden con algunos estudios(16), donde no encontraron diferencia $(P>0.05)$ en unidades Haugh al emplear $0,5,7.5$ y $10 \%$ de inclusión de PC genéticamente no mejorada. También se ha reportado(14) que la inclusión de 0, 5, 10 y $15 \%$ no afectó las unidades Haugh, ni el grosor del cascarón. Riyazi et $a(10)$, observaron que al utilizar $0,5,10 \mathrm{y}$ $15 \%$ de $\mathrm{PC}$ en dietas para gallinas $\mathrm{Hy}$-Line W36 no se afectó significativamente ( $P>0.05)$ las unidades Haugh, ni el grosor del cascarón en cualquier nivel de inclusión.

Los resultados obtenidos en la prueba sensorial para color de la yema y sabor del huevo en el Exp 1, indicaron un efecto significativo $(P<0.01)$ entre tratamientos con una mayor aceptación al sabor en los tratamientos con 0 y $13.2 \%$ de PC respecto a las gallinas alimentadas con $26.4 \%$ de inclusión de dicha pasta. Estos resultados coinciden en parte con otros investigadores(11), quienes realizaron una evaluación sensorial del huevo de gallinas Bovans Brown con una dieta 0, 4, 6 y $8 \%$ inclusión de PC, clasificándolo en una escala de 4 puntos en rango de 2 a 5 por el grado de gusto ( 2 inaceptable, 3 aceptable, 4 bueno y 5 muy bueno). Los huevos de gallinas alimentadas con el $8 \%$ se calificaron como de sabor inferior $(P<0.05)$ respecto a los otros tratamientos. También en un estudio de prueba sensorial empleando huevos hervidos(14), encontraron diferencia $(P<0.05)$ en sabor y olor entre la dieta control $0 \%$ y 8 a $10 \%$ de inclusión. Por otro lado, difiriendo con estos resultados, se indica(7), que al incluir 12 y $20 \%$ de PC en dietas para gallinas $\mathrm{Hy}$-line Brown se produjo un incremento en el olor a pescado en los to determine how much sinapine is needed to cause this phenomenon, it was found that inclusion of more than $1 \mathrm{~g}$ sinapine per kilogram feed in diets for semi-heavy hens will cause eggs to have this undesirable odor and flavor(20).

Yolk color was unaffected and no fishy odor or flavor was reported in eggs from Exp 2, in which the highest inclusion was $13.2 \%$. The yolk color results agree with a study in which ISA Brown and White Leghorn hens were fed diets containing 10,15 or $20 \%$ canola(8); no changes in color perception were noted in any of the treatments. This study did not include flavor evaluations.

\section{CONCLUSIONS AND IMPLICATIONS}

Inclusion of canola meal at three levels (6.6, 13.2 and $26.4 \%$ ) in replacement of the protein $(25,50$ and $90 \%)$ in soybean meal in isoproteic and isocaloric diets fed ISA Brown hens had no effect on performance, Haugh Units values, yolk color and shell thickness. However, replacement of $90 \%$ of the soybean meal protein $(26.4 \%$ canola meal replacement level) imparted a fishy odor to the eggs.

End of english version

huevos crudos y cocidos. Estos mismos autores encontraron que el sabor a pescado en huevos cocidos fue numéricamente más bajo que en huevos crudos. Por otro lado, se sabe que la sinapina es la forma predominante de la colina en $\mathrm{PC}$, precursor de la trimetilamina, que puede producir el olor y sabor a pescado en los huevos que proceden de gallinas semipesadas alimentadas con $\operatorname{PC}(17,18,19)$. En un experimento(20), determinaron que la inclusión de más de $1 \mathrm{~g}$ de sinapina por kilogramo de alimento en dietas para gallinas semipesadas, causa que los huevos obtengan un olor y sabor a pescado. 
En el Exp 2 de este estudio, no se afectó la percepción del color ni el sabor en el huevo con la inclusión de 6.6 y $13.2 \%$ de PC; parte de estos resultados coinciden con los obtenidos por otros investigadores(8), quienes utilizaron 10,15 y $20 \%$ de PC en dietas para gallinas Isa-Brown y White Leghorn donde participaron tres operadores experimentados, y en ambas estirpes de aves no se mostró un efecto detrimental en la percepción del color de la yema en cualquier nivel de PC empleada en la dieta; sin embargo, estos autores no evaluaron la percepción del sabor para poder comparar los resultados de esta variable con los obtenidos en esta investigación.

\section{CONCLUSIONES E IMPLICACIONES}

Con base a los resultados obtenidos en el presente estudio en gallinas Isa Brown en semilibertad alimentadas con dietas isoproteícas e isocalóricas sorgo-soya, con inclusión de pasta de canola $(0.0,6.6,13.2$ y 26.4), como reemplazo de la proteína $(0.0,25,50$ y $90 \%)$ de pasta de soya, no se afectó el rendimiento productivo, unidades Haugh, color de la yema y grosor de cascarón. Sin embargo, el reemplazo del $90 \%$ impartió sabor a pescado al huevo marrón.

\section{LITERATURA CITADA}

1. ASERCA/SAgARPA. Apoyos y Servicios de Comercialización Agropecuaria/Secretaría de Agricultura, Ganadería, Desarrollo Rural, Pesca y Alimentación. Pasta y Harina de Soya. Revista Claridades Agropecuarias 1994;8:14-22.

2. Leeson S, Summers JD. Commercial poultry nutrition. $3^{\text {rd }}$ ed. Ontario, Canada: University books; 2005.

3. Newkirk RW. Canola meal feed industry guide. 4th ed. Winnipeg, Canada: Publication of Canola Council of Canada. 2009.

4. Simbaya J, Slominski BA, Rakow G, Campbell LD, Downey RK, Bell JM. Quality characteristics of yellow seeded Brassica seed meals: Protein, carbohydrates, and dietary fiber components. J Agric Food Chem 1995;43:2062-2066.
5. Slominski BA, Jia W, Mikulski D, Rogiewiez A, Jankowski J, Rakow G, et al. Chemical composition and nutritive value of low-fiber yelow-seeded $B$ napus and $B$. juncea canola for poultry. Int Rapeseed Cong. Prague. 2011:443-445.

6. Ciurescu GT. Efficiency of soybean meal replacement by rapeseed meal and/or canola seeds in commercial layer diets. Archiva Zootechnica 2009;12:27-33.

7. Bell GE, Nottingham SM, Perez-Maldonado RA. Sensory evaluation of egg from hens fed on canola meal and cottonseed meal based diets. Proc Aust Poult Sci Symp 2002;14:100.

8. Perez-Maldonado RA, Barram KM. Evaluation of australlian canola meal for production and egg quality in two layer strains. Proc Aust Poult Sci Symp 2004;16:171-174.

9. Lichounikova $M$, Zerman $L$, Jandasek J. The effect on feeding untreated rapessed and iodine suplement on egg quality. Anim Sci 2008;53(2):77-82.

10. Riyazi SR, Ebrahimnezhad $Y$, Nazeradl K, Maheri-Sis N, Salamatdust $R$, Vahdatpour $T$. The effects of replacing soybean meal with different levels of rapeseed meal on egg quality characteristics of commercial laying hens. Asian J Anim Vet Adv 2009;4:337-341.

11. Swiatkiewicz S, Koreleski J, Srczewska-Wlosek A. Egg performance, egg quality, and nutrient utilization in laying hens fed diets with different levels of rapeseed expeller cake. Agric Food Sci 2010;19:233-239.

12. SPSS Inc. SPSS for Windows Version 17.0, 2014.

13. Campbell LD, Slominski BA, Falk KC, Wang Y. LowGlucosinolate canola in laying hen diets [abstract]. Proc 10th Int Rapeseed Cong. Camberra, Australia. 1999:276.

14. Lichovnikova M, Zerman L, Kracmar S, Klecker D. The effect of the extrusion process on the digestibility of feed given to laying hens. Anim Feed Sci Technol 2004;116:313-318.

15. Rojas RE, Ávila GE, Tirado AJ. El valor nutritivo de la harina de canola en el comportamiento de pollos de engorda y gallinas en postura. Tec Pecu Méx 1985;49:135-142.

16. Olomu JM, Robblee AR, Clandinin DR, Hardin RT. Effects of Span rapeseed meal on productive performance, egg quality, composition of liver and hearts and incidence of "fatty livers" in laying hens. Can J Anim Sci 1975;55:71-75.

17. Qiao HY, Dahiya JP, Classen HL. Nutritional and physiological effects of dietary sinapic acid (4-hydroxy-3,5-dimethoxycinnamic acid) in broiler chickens and its metabolism in the digestive tract. Poult Sci 2008;87:719-726.

18. Ward AK, Classen HL, Buchanan FC. Fishy-egg tainting is recessively inherited when brow-shelled layers are fed canola meal. Poult Sci 2009;88:714-721.

19. Johnson ML, Dahiya JP, Olkowski AA, Classen HL. The effect of dietary sinapic acid (4-hydroxy-3, 5-dimethoxy-cinnamic acid) on gastrointestinal tract microbial fermentation, nutrient utilization, and egg quality in laying hens. Poult Sci 2008;87:958-963.

20. Goh, YK, Clandinin DR, Robblee AR, Darlington, K. The effect of level of sinapine in a laying ration on the incidence of fishy odor in eggs from brown-shelled egg layers. Can J Anim Sci 1979;59:313-316. 